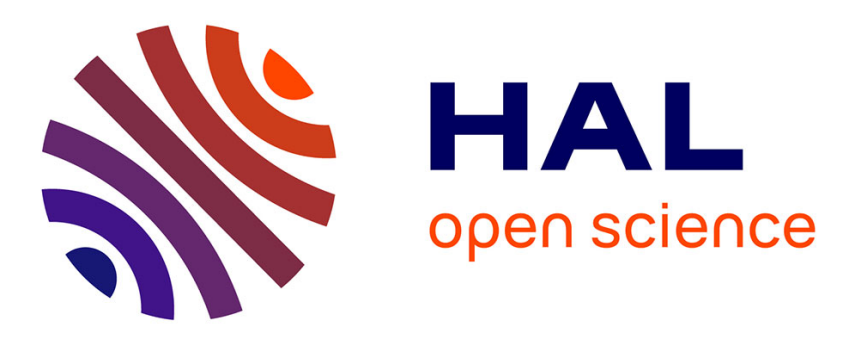

\title{
Delay-hybrid-dependent stability for systems with large delays
}

Xi-Ming Sun, Xue-Fang Wang, Frédéric Mazenc

\section{To cite this version:}

Xi-Ming Sun, Xue-Fang Wang, Frédéric Mazenc. Delay-hybrid-dependent stability for systems with large delays. IEEE transactions on systems, man, and cybernetics, 2018, pp.1-12. hal-01849022

\section{HAL Id: hal-01849022 \\ https://hal.inria.fr/hal-01849022}

Submitted on 25 Jul 2018

HAL is a multi-disciplinary open access archive for the deposit and dissemination of scientific research documents, whether they are published or not. The documents may come from teaching and research institutions in France or abroad, or from public or private research centers.
L'archive ouverte pluridisciplinaire HAL, est destinée au dépôt et à la diffusion de documents scientifiques de niveau recherche, publiés ou non, émanant des établissements d'enseignement et de recherche français ou étrangers, des laboratoires publics ou privés. 


\title{
Delay-hybrid-dependent stability for systems with large delays
}

\author{
Xi-Ming Sun, Xue-Fang Wang, Frédéric Mazenc
}

\begin{abstract}
This paper investigates a problem of stability analysis for a class of nonlinear systems with a time-varying delay taking both large and small values in an alternating manner. This problem is a delay-dependent stability analysis one but it shares some features with delay-independent ones. Hence traditional stability analysis techniques for systems with delay do not apply. By building upon our previous works, we first introduce the concept of delay-hybriddependent stability, which grasps the features of the delays described above. To ease the problem we investigate, we represent the studied system as a system with a switched delay. Then by using switching techniques and LyapunovKrasovskii functionals (LKFs), we provide a new stability criterion. Next, in the linear context, we show how the needed LKFs can be constructed by developing an original design of LKFs.
\end{abstract}

\section{Index Terms}

Stability, Large delays, Switching, Lyapunov-Krasovskii functional.

\section{INTRODUCTION}

Time-delays are unavoidable in many practical control systems such as, networked control systems (NCSs) [1], temperature control systems [2], multi-agent systems [3], [4], sampled control systems [5], biological systems [6], pneumatic systems [7]. The presence of time-delays often leads to poor performances and even instability [8]. Consequently, the stability analysis of time-delay systems has attracted considerable attention in last two decades (see, for example, [9]-[15] and references cited therein) in both the delay-independent and delay-dependent stability context. It is well-known that delay-dependent stability conditions are in general less conservative than delayindependent ones, especially for systems with small delays. Let us recall that the stability of a system is called delay-independent if this system is stable no matter what the size of its delay is and it is called delay-dependent if the stability property holds for delays belonging to specific intervals only. Thus, the maximum allowable size of the delay is a fundamental parameter for assessing the conservatism of a delay-dependent stability criterion. It is worth observing that many works have been done to reduce the conservatism of delay-dependent stability results for time-delay systems (see, for example, [3], [4], [16]-[22]).

However, with the development of NCSs [23], [24], uncertain kinematics systems [25], [26], fault tolerant control [27], [28], and controller/actuator failure systems [29], [30], a new class of time-delay systems, whose stability cannot be established by the traditional stability criteria due to the presence of time-varying delays, has to be considered. For instance, in NCSs, due to the effect of the consecutive packet dropouts and the application of zero-order hold, in some local time periods, there will appear large delays (see, for example [31]) whose upper bound may be larger than the maximum allowable constant delay that is deduced from classical delay-dependent stability criteria [8], [10], [32]. In the research of controller/actuator failure problems, the controller or actuator failure may lead to locally large delay periods [30]. In addition, with the development of the Internet of things, the large delays may appear during the process of the data transmission to the terminal (see, for example, [33]). Hence, it is of both theoretical and practical significance to investigate this kind of system model and make an analysis of such systems. In fact, some results of such systems has been successfully applied to multi-agent systems (see, for

X. M. Sun, X. F. Wang are with the School of Control Science and Engineering, Dalian University of Technology, Dalian 116024, P. R. China (email: sunxm@dlut.edu.cn,xfwang2015@mail.dlut.edu.cn).

Corresponding author: X. M. Sun. Tel.: +86 411 84707580; fax: +86 41184707581.

This work were supported by the National Natural Science Foundation of China under Grant 61325014 and the PHC Bosphore 2016. Title: Switched nonlinear and time-varying systems with arbitrarily long delay.

F. Mazenc is with EPI Inria-Saclay DISCO, Laboratoire des Signaux et Systèmes, CNRS-CentraleSupélec, 3 rue Joliot Curie, 91192 Gif-sur-Yvette, France (email: frederic.mazenc@12s.centralesupelec.fr (Frederic Mazenc)). 
example, [4]). Indeed, the delay of these systems presents a very specific property: roughly speaking, in some time intervals, it is small and in others it is large. The stability of these systems is not surprising: the intuition suggests that if a system is stable when its delay is small, then if its delay is small in sufficiently long and frequent time intervals, the systems may be stable, no matter how large is the delay in some other intervals. This motivates the present paper where a new stability analysis for systems of this type is proposed.

For a system with a delay that is sometimes large and sometimes small, we call the two different delay periods large delay periods (LDPs) and small delay periods (SDPs) and we call the system itself system with mixed delay periods.

The key ideas of our new result are the following. First, we observe that the change from one delay period to the other can be described as a switching behavior. By representing the delay as a switched delay, we represent a system with mixed delay periods as a switched system and call the stability of such a system delay-hybrid-dependent stability. This crucial new idea will enable us to study the stability of these systems by resorting to the switched system theory, for which several efficient tools are available (for more information on switched systems, see for instance [34]-[36] and references cited therein). It turns out that the switched system we obtain is composed of a stable and an unstable subsystems. The fundamental idea we will use to study this system consists in obtaining for the solutions a decay rate estimate for the stable subsystem, an increase rate estimate of the solutions for the unstable one and next to use these estimates to deduce the stability of the system under assumptions pertaining to the switching sequences (basically a restriction on the frequency and the length of LDPs is imposed).

The present paper can be seen as a review, comprehensive extension and simplification of our previous works. Let us explain how. We have done some related works [37]-[39] to deal with the presence of large delays. In [37], the large delay sequence concept was firstly introduced for analyzing the stability of discrete-time systems with time-delays and the normal delay system was first turned to a class of switched delay system. Then, the concepts of the length rate and frequency of the large delay period were extended to linear continuous system in [38]. Furthermore, the concept of the large delay period was extended to nonlinear delay systems to establish an Input-to-State Stability (ISS) property in [39]. Let us observe that the methods used in these contributions to construct Lyapunov functionals and to analyze the stability of systems with large delay are rather complex in papers mentioned above [37]-[39]. In addition, the given stability conditions are all based on LMI forms which cannot incarnate the inherent features of Lyapunov functions and the computation on LMIs may be a little bit complex. To obtain simpler results, in the present paper, for the first time, we propose the delay-hybrid-dependent stability notion, which is different from the classical delay-dependent and delay-independent stability notions. In certain applications, the new stability concept is very helpful, such as networked controlled systems, the standard delay-dependent and delay-independent analysis are too restrictive. This is because the delay signal might have large peaks, while the frequency of these large peaks may be very low. In such scenarios, the usage of the delayhybrid-dependent stability notion might be helpful in reducing the conservatism in the results. This new stability concept can be viewed as the summarization of our previous stability definitions since in our previous works, we in fact gave some examples related to large delays and some analysis results for the large delayed system but did not propose the delay-hybrid-dependent stability criterion clearly. Moreover, with a view to further studies, pertaining for example to controller designs, we give a constructed Lyapunov-Krasovskii functional technique in the paper to conveniently check the delay-hybrid-dependent stability, which is never used in the analysis of large delayed case before although some of techniques may be used to analyze the small delay case. Besides, for a class of linear systems $\dot{x}(t)=A x(t)+B x\left(t-d_{\sigma(t)}(t)\right)$, the designed Lyapunov-Krasovskii functional technique proposed in the present paper does not assume that the matrix $A$ is Hurwitz, which is more efficient in practice compared with our previous works. So such a technique gives a relaxed condition compared with our previous works given in LMI forms, and may be more easier to utilize or extend the delay-hybrid-dependent stability. Finally, we illustrate our theoretical results with two examples.

The remainder of this paper is organized as follows. Section II introduces the problem and some definitions. Section III gives the main results. Section IV demonstrates the effectiveness of the proposed method through two examples. Finally, in Section V we draw some concluding remarks.

\section{A. Notation}

The argument of the functions will be omitted whenever no confusion can arise from the context. For a vector $x \in \mathbb{R}^{n}$, the symbol $|\cdot|$ denotes the Euclidean norm of $x . P>0$ is used to denote a symmetric positive definite 
matrix $P \in \mathbb{R}^{n \times n}$ and $\lambda_{\min }(P)$ denotes the minimum eigenvalue of $P . L_{2}\left([-\tau, 0] ; \mathbb{R}^{n}\right)$ is used to denote the space of the square integrable functions $\phi:[-\tau, 0] \rightarrow \mathbb{R}^{n}$. For any function $\phi \in C^{0}\left([-\tau, 0], \mathbb{R}^{n}\right)$, we let $|\phi|=\sup _{\theta \in[-\tau, 0]}|\phi(\theta)|$.

For a given fixed time-delay $\tau$, we define $x_{t}(\theta)=x(t+\theta)(\theta \in[-\tau, 0])$. The space of functions $W[-\tau, 0]$ denotes the Banach Space of absolutely continuous functions $\phi:[-\tau, 0] \rightarrow \mathbb{R}^{n}$ with $\dot{\phi} \in L_{2}\left([-\tau, 0] ; \mathbb{R}^{n}\right)$, equipped with the norm

$$
|\phi|_{W}=\max _{\theta \in[-\tau, 0]}|\phi(\theta)|+\left[\int_{-\tau}^{0}|\dot{\phi}(s)|^{2} d s\right]^{\frac{1}{2}} .
$$

For a given fixed time-delay $\tau$, we define $x_{t}(\theta)=x(t+\theta)(\theta \in[-\tau, 0])$. We end this section with a definition of derivative for functionals, which will be instrumental in the forthcoming Lyapunov analyzes.

Let $V(t, \phi, \varphi): \mathbb{R}_{\geq 0} \times E_{\text {in }} \rightarrow \mathbb{R}^{+}$with $E_{\text {in }}=W[-\tau, 0] \times L_{2}\left([-\tau, 0], \mathbb{R}^{n}\right)$ be a continuous functional. Define (see, e.g., [40])

$$
\dot{V}(t, \phi, \dot{\phi})=\limsup _{h \rightarrow 0^{+}} \frac{V\left(t+h, x_{t+h}(t, \phi), \dot{x}_{t+h}(t, \phi)\right)-V(t, \phi, \dot{\phi})}{h},
$$

where $x_{t}\left(t_{0}, \phi\right)$, for $t \geq t_{0}$, is a solution with the initial condition $x_{t_{0}}=\phi \in W$.

\section{PROBLEM FORMULATION}

\section{A. The studied systems}

We consider the nonlinear system:

$$
\dot{x}(t)=f(x(t), x(t-d(t)))
$$

where $x \in \mathbb{R}^{n}$ is the state vector, $f: \mathbb{R}^{n} \times \mathbb{R}^{n} \rightarrow \mathbb{R}^{n}$ is of class $C^{1}$ with $f(0,0)=0, d(t)$ denotes nonnegative time-varying delay. Along the paper, we let system (2) satisfy the following assumption:

Assumption 1: The function $d(t)$ is piecewise- $C^{1}$ and there are constants $d^{\sharp} \geq 0$ and $d_{b}>0$ such that $|\dot{d}(t)| \leq d^{\sharp}$ almost everywhere and $d(t) \leq d_{b}$ for all $t \geq 0$. There is a constant $h \in\left[0, d_{b}\right)$ and a sequence $\left(t_{i}\right)$ such that $0 \leq t_{0}<t_{1}<t_{2}<\cdots$ and for all $t \in\left[t_{2 j}, t_{2 j+1}\right)$,

$$
d(t) \leq h
$$

Moreover, there is $v>0$ such that for all $i \in \mathbb{N}, t_{i+1}-t_{i} \geq v$.

Remark 1: The case where it is assumed that $d(t)>h$ when $t \in\left[t_{2 j+1}, t_{2 j+2}\right)$ is entirely similar to the one of Assumption 1 and so we will not consider it.

We define for later use two sets:

$$
\mathscr{E}_{S}=\bigcup_{i=0}^{+\infty}\left[t_{2 i}, t_{2 i+1}\right) \text { and } \mathscr{E}_{l}=\bigcup_{i=0}^{+\infty}\left[t_{2 i+1}, t_{2 i+2}\right)
$$

and we recall the following definitions:

Definition 1: [38] Under Assumption 1, the time interval $\left[t_{2 j}, t_{2 j+1}\right)$ for any $j \in \mathbb{N}$ is called small delay period and the time interval $\left[t_{2 j+1}, t_{2 j+2}\right)$ for any $j \in \mathbb{N}$ is called large delay period.

We introduce two definitions:

Definition 2: [38] For any $T_{2}>T_{1} \geq 0, T_{l d p}\left(T_{1}, T_{2}\right)$ denotes the total time length of LDPs in time interval $\left[T_{1}, T_{2}\right)$ and $T_{s d p}\left(T_{1}, T_{2}\right)$ denotes the total time length of SDPs in $\left[T_{1}, T_{2}\right)$.

Definition 3: For any $T_{2}>T_{1} \geq 0, N_{s}\left(T_{1}, T_{2}\right)$ denotes the number of $t_{i}$ belonging to $\left(T_{1}, T_{2}\right)$.

Definition 4: We say that system (2) is a system with mixed delay periods when the delay is defined over the interval $\left[t_{0},+\infty\right)$ and operates in an alternating manner between SDPs and LDPs. The stability of such a system shares features with the delay-dependent and delay-independent stability and this stability property is called delayhybrid-dependent stability and we say that the system is delay-hybrid-dependently stable.

Next based on the above description and definitions, hybrid representation of the studied systems is given. 


\section{B. Hybrid representation of the system}

When LDPs and SDPs occur in alternative way in system (2), switching techniques can be adopted to analyze its delay-hybrid-dependent stability properties. To do this, we rewrite system (2) as the following switched system:

$$
\begin{aligned}
\dot{x}(t) & =f\left(x(t), x\left(t-d_{\sigma(t)}(t)\right)\right) \\
x(\theta) & =\phi(\theta), \quad \theta \in\left[-d_{b}, 0\right]
\end{aligned}
$$

where $\phi$ denotes a continuously differentiable vector-valued initial function defined over $\left[-d_{b}, 0\right], \sigma:[0,+\infty) \rightarrow$ $M=\{1,2\}$ is a function called switching signal, defined as follows: $\sigma(t)=1$ when $t \in \mathscr{E}_{s}$ and $\sigma(t)=2$ when $t \in \mathscr{E}_{l}$ and $d(t):=d_{1}(t)$ when $t \in \mathscr{E}_{S}$ and $d(t):=d_{2}(t)$ when $t \in \mathscr{E}_{l}$. Thus $d(t):=d_{\sigma(t)}(t)$ for all $t \geq 0$, which implies that system (5) is identical to system (2).

In next section, stability criteria with some restrictions on the time length and the number of occurrence of large delays of nonlinear and linear systems are given.

\section{DELAY-HYBRID-DEPENDENT STABILITY RESULTS}

\section{A. General stability result}

We give in this section delay-hybrid-dependent stability conditions for the general nonlinear system (5): we are ready to state and prove a stability result which, basically, shows that the exponential stability of system (5) is guaranteed under certain restrictions on the length and the frequency of the LDPs. Before giving the main theorem, we first present some conditions:

$C_{1}:$ The inequalities

$$
\gamma_{1}|\phi(0)|^{2} \leq V_{j}(t, \phi, \dot{\phi}) \leq \gamma_{2}|\phi|_{W}^{2}, j=1,2
$$

and

$$
V_{1}(t, \phi, \dot{\phi}) \leq \mu V_{2}(t, \phi, \dot{\phi}), \quad V_{2}(t, \phi, \dot{\phi}) \leq \mu V_{1}(t, \phi, \dot{\phi})
$$

hold for all $t \geq 0$ and $\phi \in W[-\tau, 0]$.

$C_{2}$ : The derivatives of $V_{1}$ and $V_{2}$ along the trajectories of (5) satisfy

$$
\begin{aligned}
& \dot{V}_{1}(t, \phi, \dot{\phi}) \leq-\alpha_{1} V_{1}(t, \phi, \dot{\phi}), \quad \forall t \in \mathscr{E}_{S} \\
& \dot{V}_{2}(t, \phi, \dot{\phi}) \leq \alpha_{2} V_{2}(t, \phi, \dot{\phi}), \quad \forall t \in \mathscr{E}_{l}
\end{aligned}
$$

$C_{3}$ : Let

$$
R\left(t_{0}, t\right)=-\alpha_{1} T_{s d p}\left(t_{0}, t\right)+\alpha_{2} T_{l d p}\left(t_{0}, t\right) .
$$

There are $\alpha_{*}>0, \beta_{*}>0, \eta_{i}>0, i=1,2$ such that for all $t \geq t_{0}$, the inequalities

$$
R\left(t_{0}, t\right) \leq-\alpha_{*}\left(t-t_{0}\right)+\eta_{1}, \quad N_{s}\left(t_{0}, t\right) \leq \beta_{*}\left(t-t_{0}\right)+\eta_{2}
$$

are satisfied.

$C_{4}$ : The inequality

$$
\ln (\mu) \beta_{*}<\alpha_{*}
$$

is satisfied.

Theorem 1: Let system (5) satisfy Assumption 1. Assume that there are two functionals $V_{1}$ and $V_{2}$ satisfying the properties described in (1) and positive real numbers $\gamma_{i}, \alpha_{i}, i=1,2$ and $\mu \geq 1$ such that conditions (6)-(11) hold. Then for all $t \geq t_{0}$,

$$
|x(t)|^{2} \leq e^{\left(\ln (\mu) \beta_{*}-\alpha_{*}\right)\left(t-t_{0}\right)} \gamma_{*}\left|x_{t_{0}}\right|_{W}^{2}
$$

with $\gamma_{*}=e^{\eta_{1}+\ln (\mu)\left(\eta_{2}+1\right)} \frac{\gamma_{2}}{\gamma_{1}}$.

Proof: See Appendix A.

Remark 2: The fact that the inequalities in (7) need to be satisfied with the same constant $\mu$ is not restrictive since if two Lyapunov functionals $\mathscr{V}_{1}$ and $\mathscr{V}_{2}$ are such that

$$
\mathscr{V}_{1}(t, \phi, \dot{\phi}) \leq \mu_{1} \mathscr{V}_{2}(t, \phi, \dot{\phi}), \quad \mathscr{V}_{2}(t, \phi, \dot{\phi}) \leq \mu_{2} \mathscr{V}_{1}(t, \phi, \dot{\phi})
$$


then, the functionals

$$
V_{1}=\sqrt{\frac{\mu_{2}}{\mu_{1}}} \mathscr{V}_{1}, \quad V_{2}=\mathscr{V}_{2}
$$

satisfy the inequalities (7) with $\mu=\sqrt{\mu_{1} \mu_{2}}$. Moreover, (7) is just used to restrain the boundedness of $V_{1}, V_{2}$ and make sure it will not go to infinity at switching points. So it is enough to adopt the same $\mu$ on $V_{1}, V_{2}$. Although we use different $\mu$ in (7), in order to obtain exponential stability by iterative method, we need to take the maximum value among different $\mu$. Besides, for positive definite functions $V_{1}, V_{2}$, the existence of the ratio bound $\mu$ in (7) is a sufficient condition for the existence of the comparison functions $\gamma_{1}, \gamma_{2}$ in (6).

For clearity of this idea, by constructing Lyapunov functionals, in next subsection, the stability analysis of the linear system with mixed delay periods is shown.

\section{B. Illustration}

In this section, we show how Theorem 1 can be applied to linear systems with mixed delay periods by constructing LKFs satisfying conditions $C_{1}$ and $C_{2}$. For the sake of simplicity, we present a result for time invariant systems that is based on LKFs which can be constructed via rather simple calculations. More refined, but more lengthy, constructions leading to less conservative results with respect to the size of the delays in small periods can be proposed.

Consider the system

$$
\dot{x}(t)=A x(t)+A_{d} x\left(t-d_{\sigma(t)}(t)\right),
$$

where $d_{1}, d_{2}$ and $\sigma$ are the same as those in Section II-B and the matrices $A$ and $A_{d}$ are constant and such that there are a constant $c_{a}>0$ and a symmetric positive definite matrix $P$ such that

$$
P H+H^{\top} P \leq-2 c_{a} P,
$$

where $H=A+A_{d}$ and $I$ is the identity matrix. To simplify the calculations, we assume that $d_{2}$ is of class $C^{2}$ and $d^{\sharp}<1$ and $\left|\ddot{d}_{2}(t)\right| \leq \bar{d}$ with $\bar{d}=\left[2|P A|+\frac{1}{4}|A|^{2}+\left|P A_{d}\right|+\frac{\left|P A_{d}\right|+\frac{1}{4}\left|A_{d}\right|^{2}}{1-d^{\sharp}}\right]\left(1-d^{\sharp}\right)^{2}$. Moreover, we assume that

$$
h \leq \min \left\{1, \frac{1}{4 \sqrt{c_{b}}}, \frac{c_{a} \lambda_{\min }(P)}{\sqrt{16 c_{b}|H|^{2}}}, \frac{1}{\sqrt{8\left|A_{d}\right|^{2}}}, \frac{1}{3 c_{a}}\right\}
$$

with $c_{b}=\frac{\left|A_{d}^{T} P A_{d}\right|}{c_{a}}$.

Remark 3: A crucial aspect of the family we consider is that we do not assume that the matrix $A$ is Hurwitz, which is frequently encountered in practice and implies that in general this family is not delay independent stable.

Now, we show how we can construct Lyapunov functionals ensuring that the conditions $C_{1}$ and $C_{2}$ of Theorem 1 are satisfied by system (13).

First step. Let us study system (13) when $t \in \mathscr{E}_{s}$. Let

$$
v_{1}(x)=x^{\top} P x .
$$

Since

$$
\dot{x}(t)=H x(t)-A_{d} \int_{t-d_{1}(t)}^{t} \dot{x}(\ell) d \ell,
$$

the derivative of $v_{1}$ along (17) satisfies

$$
\begin{aligned}
\dot{v}_{1}(t) & \leq-2 c_{a} x(t)^{\top} P x(t)-2 x(t)^{\top} P A_{d} \int_{t-d_{1}(t)}^{t} \dot{x}(\ell) d \ell \\
& \leq-c_{a} x(t)^{\top} P x(t)+\frac{1}{c_{a}}\left|A_{d}^{T} P A_{d}\right|\left|\int_{t-d_{1}(t)}^{t} \dot{x}(\ell) d \ell\right|^{2} \\
& \leq-c_{a} x(t)^{\top} P x(t)+c_{b} h \int_{t-h}^{t}|\dot{x}(\ell)|^{2} d \ell .
\end{aligned}
$$


Let us introduce the operator

$$
v_{2}\left(x_{t}, \dot{x}_{t}\right)=v_{1}(x(t))+2 c_{b} h \int_{t-h}^{t} \int_{m}^{t}|\dot{x}(\ell)|^{2} d \ell d m .
$$

Then

$$
\begin{aligned}
\dot{v}_{2}(t) & \leq-c_{a} v_{1}(x(t))-c_{b} h \int_{t-h}^{t}|\dot{x}(\ell)|^{2} d \ell+2 c_{b} h^{2}\left|H x(t)-A_{d} \int_{t-d_{\sigma(t)}(t)}^{t} \dot{x}(\ell) d \ell\right|^{2} \\
& \leq-c_{a} v_{1}(x(t))-c_{b} h \int_{t-h}^{t}|\dot{x}(\ell)|^{2} d \ell+4 c_{b} h^{2}\left[|H|^{2}|x(t)|^{2}+\left|A_{d}\right|^{2} h \int_{t-h}^{t}|\dot{x}(\ell)|^{2} d \ell\right] .
\end{aligned}
$$

From (16), it follows that

$$
\dot{v}_{2}(t) \leq-c_{a} v_{1}(x(t))-c_{b} h \int_{t-h}^{t}|\dot{x}(\ell)|^{2} d \ell+4 c_{b} h^{2}\left[\frac{|H|^{2}}{\lambda_{\min }(P)} v_{1}(x(t))+h\left|A_{d}\right|^{2} \int_{t-h}^{t}|\dot{x}(\ell)|^{2} d \ell\right] .
$$

Since (15) implies that

$$
\frac{16 c_{b} h^{2}|H|^{2}}{\lambda_{\min }(P)} \leq c_{a} \text { and } \quad 8 h^{2}\left|A_{d}\right|^{2} \leq 1
$$

the inequality

$$
\dot{v}_{2}(t) \leq-\frac{3 c_{a}}{4} v_{1}(x(t))-\frac{c_{b}}{2} h \int_{t-h}^{t}|\dot{x}(\ell)|^{2} d \ell
$$

is satisfied. Noticing that

$$
\frac{1}{2 c_{b} h^{2}}\left(v_{2}\left(x_{t}, \dot{x}_{t}\right)-v_{1}(x(t))\right) \leq \int_{t-h}^{t}|\dot{x}(\ell)|^{2} d \ell
$$

we deduce that

$$
\dot{v}_{2}(t) \leq-\frac{3 c_{a}}{4} v_{1}(x(t))-\frac{1}{4 h} 2 c_{b} h \int_{t-h}^{t} \int_{m}^{t}|\dot{x}(\ell)|^{2} d \ell d m .
$$

Therefore

$$
\dot{v}_{2}(t) \leq-3 c_{c} v_{2}\left(x_{t}, \dot{x}_{t}\right)
$$

with $c_{c}=\frac{c_{a}}{4}$ because $h \leq \frac{1}{3 c_{a}}$.

Second step. Now, let us study system (13) when $t \in \mathscr{E}_{l}$. Immediate calculations give

$$
\begin{aligned}
\dot{v}_{2}(t) & \leq 2 x(t)^{\top} P\left[A x(t)+A_{d} x\left(t-d_{2}(t)\right)\right]+2 c_{b} h^{2}|\dot{x}(t)|^{2} \\
& \leq 2 x(t)^{\top} P\left[A x(t)+A_{d} x\left(t-d_{2}(t)\right)\right]+2 c_{b} h^{2}\left|A x(t)+A_{d} x\left(t-d_{2}(t)\right)\right|^{2} \\
& \leq\left(2|P A|+4 c_{b} h^{2}|A|^{2}+\left|P A_{d}\right|\right)|x(t)|^{2}+\left(\left|P A_{d}\right|+4 c_{b} h^{2}\left|A_{d}\right|^{2}\right)\left|x\left(t-d_{2}(t)\right)\right|^{2}
\end{aligned}
$$

Since $h \leq \frac{1}{4 \sqrt{c_{b}}}$, the inequality

$$
\dot{v}_{2}(t) \leq c_{d} v_{1}(x(t))+c_{e} v_{1}\left(x\left(t-d_{2}(t)\right)\right)
$$

with $c_{d}=\frac{2|P A|+\frac{1}{4}|A|^{2}+\left|P A_{d}\right|}{\lambda_{\min }(P)}$ and $c_{e}=\frac{\left|P A_{d}\right|+\frac{1}{4}\left|A_{d}\right|^{2}}{\lambda_{\min }(P)}$, is satisfied.

Let

$$
v_{3}\left(t, x_{t}, \dot{x}_{t}\right)=v_{2}\left(x_{t}, \dot{x}_{t}\right)+\frac{c_{e}}{1-\dot{d}_{2}(t)} \int_{t-d_{2}(t)}^{t} v_{1}(x(m)) d m
$$

Then

$$
\begin{aligned}
\dot{v}_{3}(t) & \leq c_{d} v_{1}(x(t))+\frac{c_{e}}{1-\dot{d}_{2}(t)} v_{1}(x(t))+\frac{c_{e} \ddot{d}_{2}(t)}{\left(1-\dot{d}_{2}(t)\right)^{2}} \int_{t-d_{2}(t)}^{t} v_{1}(x(m)) d m . \\
& \leq c_{f} v_{1}(x(t))+\frac{c_{e} \bar{d}}{\left(1-d^{\sharp}\right)^{2}} \int_{t-d_{2}(t)}^{t} v_{1}(x(m)) d m \\
& \leq c_{f} v_{3}\left(t, x_{t}, \dot{x}_{t}\right)
\end{aligned}
$$

with $c_{f}=c_{d}+\frac{c_{e}}{1-d^{\sharp}}$.

Third step. It is very difficult to find or it does not exist two constants $\mu_{1}>0$ and $\mu_{2}>0$ such that $v_{2}$ and $v_{3}$ satisfy inequalities of the type (12), therefore they cannot be directly used to construction LKFs ensuring that the 
conditions $C_{1}$ and $C_{2}$ are satisfied. This leads us to construct a new LKFs in the SDPs. Let us consider again the case where $t \in \mathscr{E}_{s}$. Let

$$
v_{4}\left(t, x_{t}, \dot{x}_{t}\right)=v_{2}\left(x_{t}, \dot{x}_{t}\right)+c_{c} \int_{t-d_{2}(t)}^{t} v_{1}(x(m)) d m+\frac{c_{c}}{1+d_{b}} \int_{t-d_{2}(t)}^{t} \int_{s}^{t} v_{1}(x(m)) d m d s .
$$

Then, from (18) and the fact that $d^{\sharp}<1$, it follows that

$$
\begin{aligned}
\dot{v}_{4}(t) & \leq-2 c_{c} v_{2}\left(x_{t}, \dot{x}_{t}\right)-c_{c}\left(1-\dot{d}_{2}(t)\right) v_{1}\left(x\left(t-d_{2}(t)\right)+\frac{c_{c}}{1+d_{b}} d_{b} v_{1}(x(t))-\frac{c_{c}}{1+d_{b}}\left(1-\dot{d}_{2}(t)\right) \int_{t-d_{2}(t)}^{t} v_{1}(x(m)) d m\right. \\
& \leq-c_{c} \frac{2+d_{b}}{1+d_{b}}\left[v_{2}\left(x_{t}, \dot{x}_{t}\right)+\frac{1-d^{\sharp}}{2+d_{b}} \int_{t-d_{2}(t)}^{t} v_{1}(x(m)) d m\right] .
\end{aligned}
$$

Using

$$
v_{4}\left(t, x_{t}, \dot{x}_{t}\right) \leq v_{2}\left(x_{t}, \dot{x}_{t}\right)+c_{c} \frac{1+2 d_{b}}{1+d_{b}} \int_{t-d_{2}(t)}^{t} v_{1}(x(m)) d m,
$$

we obtain:

$$
\dot{v}_{4}(t) \leq-c_{g} v_{4}\left(t, x_{t}, \dot{x}_{t}\right)
$$

with $c_{g}=c_{c} \frac{2+d_{b}}{1+d_{b}} \min \left\{1, \frac{\left(1+d_{b}\right)\left(1-d^{\sharp}\right)}{c_{c}\left(1+2 d_{b}\right)\left(2+d_{b}\right)}\right\}$.

Now, observe that

$$
\begin{gathered}
v_{3}\left(t, x_{t}, \dot{x}_{t}\right) \leq v_{2}\left(x_{t}, \dot{x}_{t}\right)+\frac{c_{e}}{1-d^{\sharp}} \int_{t-d_{2}(t)}^{t} v_{1}(x(m)) d m, \\
v_{2}\left(x_{t}, \dot{x}_{t}\right)+c_{e} \int_{t-d_{2}(t)}^{t} v_{1}(x(m)) d m \leq v_{3}\left(t, x_{t}, \dot{x}_{t}\right), \\
v_{2}\left(x_{t}, \dot{x}_{t}\right)+c_{c} \int_{t-d_{2}(t)}^{t} v_{1}(x(m)) d m \leq v_{4}\left(t, x_{t}, \dot{x}_{t}\right) .
\end{gathered}
$$

These inequalities and (19) imply that

$$
\begin{gathered}
v_{3}\left(t, x_{t}, \dot{x}_{t}\right) \leq \mu_{1} v_{4}\left(t, x_{t}, \dot{x}_{t}\right) \quad \text { and } \\
v_{4}\left(t, x_{t}, \dot{x}_{t}\right) \leq \mu_{2} v_{3}\left(t, x_{t}, \dot{x}_{t}\right)
\end{gathered}
$$

with

$$
\begin{gathered}
\mu_{1}=\max \left\{1, \frac{c_{e}}{c_{c}\left(1-d^{\sharp}\right)}\right\} \quad \text { and } \\
\mu_{2}=\max \left\{1, \frac{c_{c}\left(1+2 d_{b}\right)}{c_{e}\left(1+d_{b}\right)}\right\} .
\end{gathered}
$$

Final Step. The conditions $C_{1}$ and $C_{2}$ of Theorem 1 are satisfied with

$$
V_{1}(t, \phi, \varphi)=\sqrt{\frac{\mu_{1}}{\mu_{2}}} v_{4}(t, \phi, \varphi), \quad V_{2}(t, \phi, \varphi)=v_{3}(t, \phi, \varphi)
$$

and $\alpha_{1}=c_{g}$ and $\alpha_{2}=c_{f}$. Indeed, for all $(\phi, \varphi) \in E_{\text {in }}$ and $t \geq 0$,

$$
\begin{aligned}
& V_{1}(t, \phi, \varphi) \leq \sqrt{\frac{\mu_{1}}{\mu_{2}}} \mu_{2} V_{2}(t, \phi, \varphi)=\mu V_{2}(t, \phi, \varphi) \\
& V_{2}(t, \phi, \varphi) \leq \mu_{1} v_{3}(t, \phi, \varphi)=\mu V_{1}(t, \phi, \varphi)
\end{aligned}
$$

with $\mu=\sqrt{\mu_{1} \mu_{2}}$.

Remark 4: From this illustration, one can know that our method is more effective in practice from four aspects:

1) It has less computational complexity.

2) The forms of Lyapunov functions are very simple and the stability criteria in present paper are easily checked.

3) It does not assume that the matrix $A$ is Hurwitz for linear system.

4) It has robustness which will be shown in the following example 2.

\section{EXAMPLES}




\section{A. Example 1}

In this section, we use an example to show the effectiveness of our results. We consider the system

$$
\dot{x}=-x\left(t-d_{\sigma(t)}(t)\right)
$$

where $x \in \mathbb{R}$ and $d_{\sigma(t)}(t)$ is a periodic function of period 20 such that $d_{1}(t)=\frac{1}{9}$ and $d_{2}(t)=d_{b}>\frac{1}{9}$ for all $t \geq 0$ and $t_{0}=0, t_{1}=p, p \in(0,20)$. We adopt the notation of the previous sections and choose

$$
v_{1}(x)=x^{2} .
$$

Then $A=0, A_{d}=-1, H=-1, d^{\sharp}=0, c_{a}=1, c_{b}=2, c_{c}=\frac{1}{4}, c_{d}=1$ and $c_{e}=\frac{5}{4}, c_{f}=\frac{9}{4}, c_{g}=\frac{2+d_{b}}{4\left(1+d_{b}\right)} \min \left\{1,4 \frac{1+d_{b}}{\left(1+2 d_{b}\right)\left(2+d_{b}\right)}\right\}$, $\mu=\sqrt{5}, \alpha_{1}=c_{g}$ and $\alpha_{2}=\frac{9}{4}$.

We have, for all $t_{0} \geq 0, t \geq t_{0}$

$$
\begin{aligned}
R\left(t_{0}, t\right) & =-c_{g} T_{s d p}\left(t_{0}, t\right)+\frac{9}{4} T_{l d p}\left(t_{0}, t\right) \\
& \leq-\alpha_{*}\left(t-t_{0}\right)+\eta_{1}
\end{aligned}
$$

with $\alpha_{*}=\frac{2+d_{b}}{4\left(1+d_{b}\right)} \min \left\{1,4 \frac{1+d_{b}}{\left(1+2 d_{b}\right)\left(2+d_{b}\right)}\right\} p-\frac{9}{4}(20-p), \eta_{1}=d_{b}$ and

$$
N_{s}\left(t_{0}, t\right) \leq \beta_{*}\left(t-t_{0}\right)+2
$$

with $\beta_{*}=\frac{1}{20}$ and $\eta_{2}=2$. We deduce from (11) that if $p$ is such that

$$
\ln (\sqrt{5}) \frac{1}{20}<\frac{2+d_{b}}{4\left(1+d_{b}\right)} \min \left\{1,4 \frac{1+d_{b}}{\left(1+2 d_{b}\right)\left(2+d_{b}\right)}\right\} p-\frac{9}{4}(20-p)
$$

then the origin of (20) is GES. This condition rewrites as

$$
\frac{\frac{\ln (5)}{40}+45}{\frac{2+d_{b}}{4\left(1+d_{b}\right)} \min \left\{1,4 \frac{1+d_{b}}{\left(1+2 d_{b}\right)\left(2+d_{b}\right)}\right\}+\frac{9}{4}}<p .
$$

Notice that when $d_{b}=\frac{1}{2}$, the system $\dot{x}=-x\left(t-d_{2}(t)\right)$ is unstable and system (20) is GES when the inequality

$$
\frac{3 \ln (5)}{320}+\frac{135}{8}<p
$$

is satisfied, which is satisfied if $17 \leq p$.

\section{B. Example 2}

In this subsection, we use the same example in [38] to compare with our previous results. Consider system (13) with

$$
A=\left[\begin{array}{cc}
-2 & 0 \\
0 & -0.9
\end{array}\right], A_{d}=\left[\begin{array}{cc}
-1 & 0 \\
-1 & -1
\end{array}\right]
$$

Here we give $\alpha_{1}=0.038, \alpha_{2}=33.8, d_{b}=10$. Choose $\alpha^{*}=0.1, \beta^{*}=0.035, c_{a}=1.899, \eta_{1}=1450, \eta_{2}=16$, then we obtain $P=\left[\begin{array}{cc}4.1802 & -2.2091 \\ * & 2.4372\end{array}\right], c_{b}=1.356, c_{c}=0.475, c_{d}=25.97, c_{e}=4.487, c_{f}=33.8, c_{g}=0.038$, $\mu_{1}=11.8, \mu_{2}=1, \mu=3.435$. From (15) we have $h \leq \min \{1,0.214,0.117,0.21,0.175\}$. The small delays and large delays are set as $d_{1}(t)=0.1|\sin (t)|$ and $d_{2}(t)=0.5|\sin (t)|+9.5$, respectively. The simulation results are shown in Fig. 1, Fig. 2 and Fig. 3. Fig. 1 shows that the considered system is exponentially stable without considering large delay case. Fig. 2 shows that system is not stable when only consider large delay case. Fig. 3 shows that, although large delays occur, the system is still exponentially stable under a certain restriction on the time length and the number of occurrence of large delays. In Fig. 3, switching signal $\sigma(t)$ is random one of some switching signals satisfying inequalities (10).

From simulation, it can be seen that $T_{l d p} \leq 42.2$ and $N_{s}=18$. It means that the $L D P$ can be permitted to occur nine times in each 100s (second) and the permitted length of $L D P$ can reach to 42.2. If choose the same parameters $\alpha_{1}=0.14, \alpha_{2}=0.3$ with [38], we can obtain the same results. However in [38], it only can be permitted to occur 


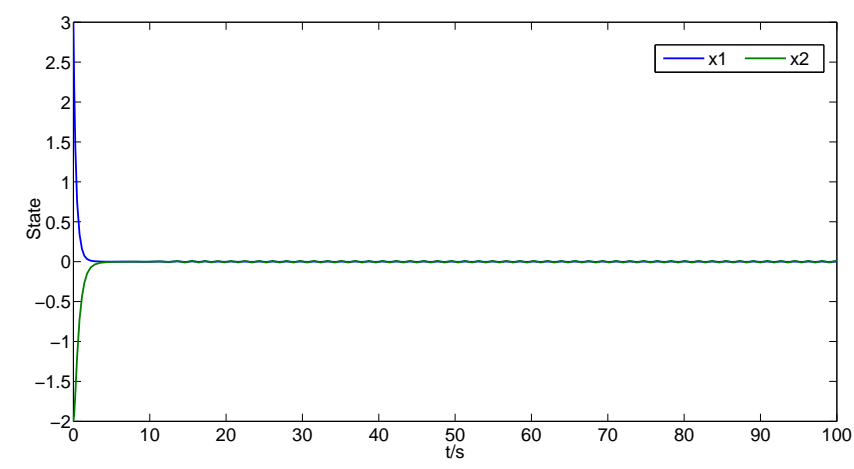

Fig. 1. State trajectories under small delay: $d_{1}(t)=0.1|\sin t|$.

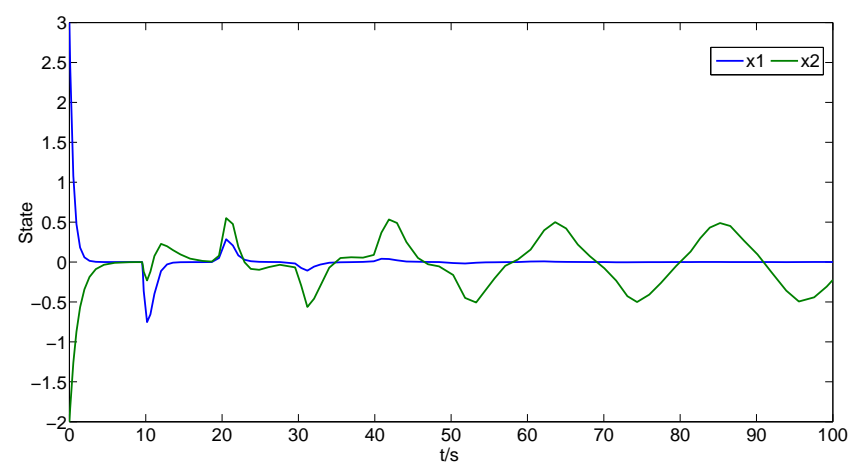

Fig. 2. State trajectories under large delay: $d_{2}(t)=9.5+0.5|\sin t|$.

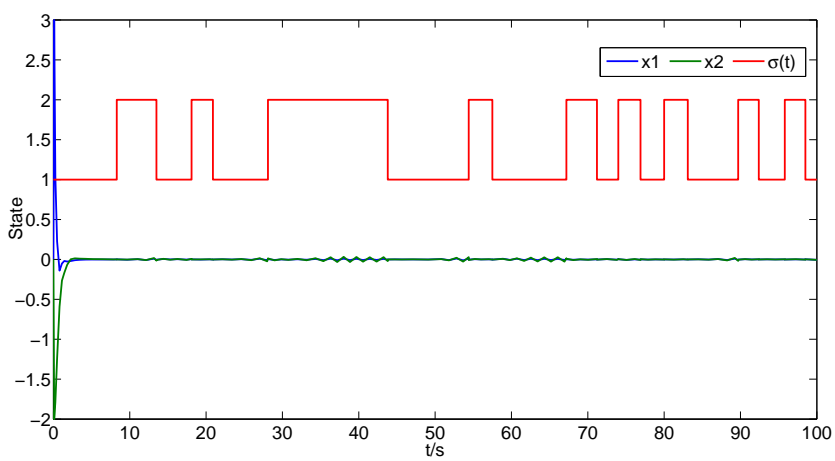

Fig. 3. State trajectories under switching signal $\sigma(t)$.

once in each 100 s and the permitted length of $L D P$ just only reach to 13.1818 . It is clearly shown the method proposed in this paper is more effective.

In order to show the robustness of our method, we consider the following system

$$
\dot{x}(t)=A x(t)+A_{d} x(t-d(t))+D \omega(t)
$$

where $A$ and $A_{d}$ are the same as the above, $D$ is constant matrix, $\omega(t)$ is the disturbance input.

In the simulation, we set $\omega(t)=0.5 e^{-0.5 t}$ and $D=\left[\begin{array}{ll}1 & 1\end{array}\right]^{T}$. The simulation results are given in Fig. 4, Fig. 5 and Fig. 6. It is clear to see from figures 4,5 and 6 that the method proposed in the present paper has certain robustness. 


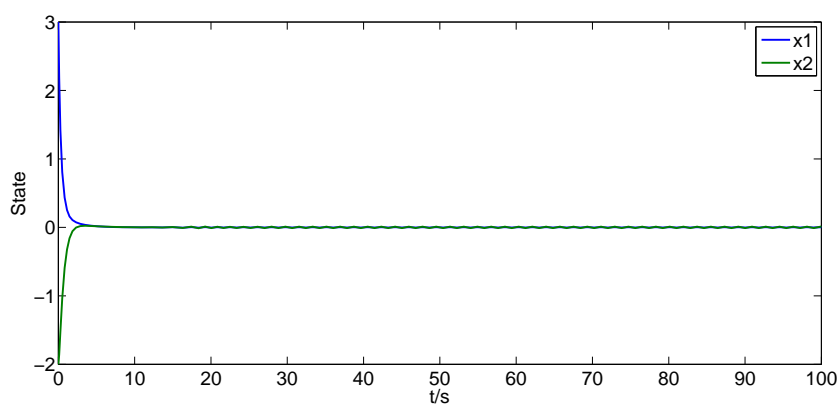

Fig. 4. State trajectories under small delay: $d_{1}(t)=0.1|\sin t|$.

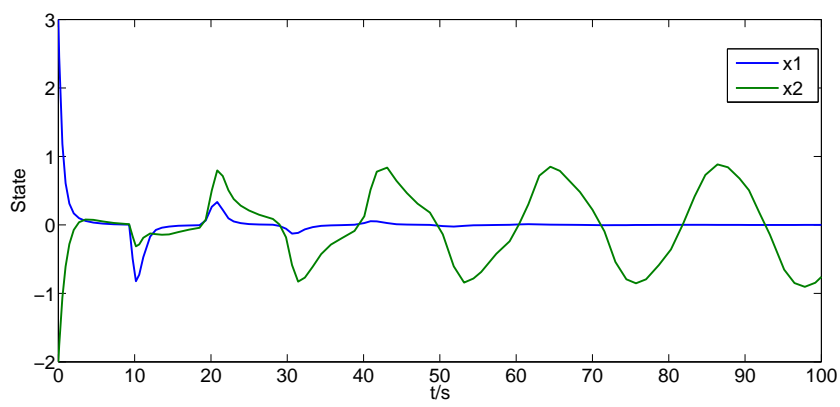

Fig. 5. State trajectories under large delay: $d_{2}(t)=9.5+0.5 \mid$ sint $\mid$.

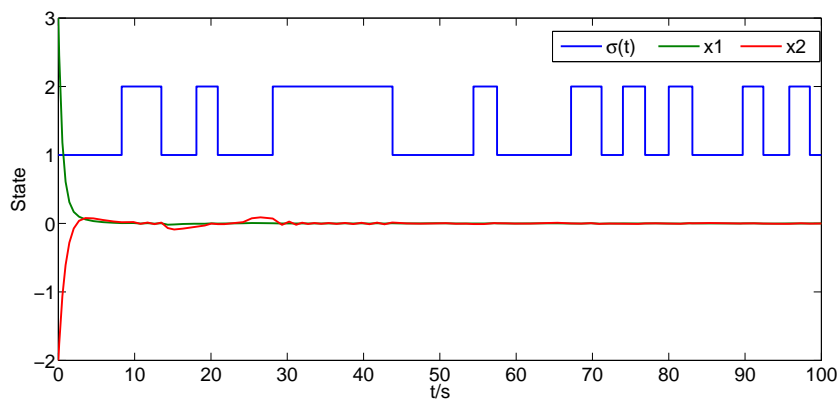

Fig. 6. State trajectories under switching signal $\sigma(t)$.

\section{CONCLUSion}

This paper has studied the stability problem of systems with a delay of a type frequently encountered in engineering problems: systems with mixed delay periods, i.e. time-varying delays with large values in small intervals and small values in long intervals. We have represented systems with this type of delay as switched systems. For them, we proposed a general stability result based on Lyapunov functionals and gave a new stability concept which is called as delay-hybrid-dependent stability. In certain applications, this new stability notion might be helpful in reducing the conservatism in the results. Numerical results have been used to illustrate the feasibility and robustness of the proposed method.

There are some remaining issues, which can be the subjects of future works, e.g., how to extend this result the stochastic delay problem (see for example, [3], [4]), how to design observer and how to propose approach for systems of neutral type in the presence of switched time-varying delays. These topics motivate our future research.

\section{APPENDIX}

First, let us observe that the properties $C_{1}$ and $C_{2}$ ensure that system (5) is forward complete. Now, based on the Lyapunov functionals $V_{1}(t, \phi, \dot{\phi})$ and $V_{2}(t, \phi, \dot{\phi})$ defined in (6), we propose a piecewise Lyapunov functional for 
system (5). Let

$$
V(t)=V_{\sigma(t)}(t, \phi, \dot{\phi})
$$

According to (8), we have

$$
V(t) \leq\left\{\begin{array}{l}
e^{-\alpha_{1}\left(t-t_{2 s}\right)} V\left(t_{2 s}\right), \quad t \in\left[t_{2 s}, t_{2 s+1}\right) \\
e^{\alpha_{2}\left(t-t_{2 s+1}\right)} V\left(t_{2 s+1}\right), \quad t \in\left[t_{2 s+1}, t_{2 s+2}\right),
\end{array}\right.
$$

for all $s \in \mathbb{N}$. Consider $t_{0} \leq t$ with $t \in\left[t_{2 j+1}, t_{2 j+2}\right)$ and $t_{0} \in\left[0, t_{2 j+1}\right)$. In view of (7) and (21), we deduce that,

$$
\begin{aligned}
V(t) & \leq e^{\alpha_{2}\left(t-t_{2 j+1}\right)} V_{2}\left(t_{2 j+1}\right) \\
& \leq e^{\alpha_{2}\left(t-t_{2 j+1}\right)} \mu V_{1}\left(t_{2 j+1}^{-}\right) \\
& \leq e^{\alpha_{2}\left(t-t_{2 j+1}\right)} \mu e^{-\alpha_{1}\left(t_{2 j+1}-t_{2 j}\right)} V_{1}\left(t_{2 j}\right) \\
& \leq \cdots \\
& \leq \mu^{N_{s}\left(t_{0}, t\right)} e^{-\alpha_{1} T_{s d p}\left(t_{0}, t\right)} e^{\alpha_{2} T_{l d p}\left(t_{0}, t\right)} V_{q}\left(t_{0}\right),
\end{aligned}
$$

where $q=1$ or 2 . Next, considering the case where $t \in\left[t_{2 j}, t_{2 j+1}\right)$, we deduce easily that for all $t \geq t_{0} \geq 0$,

$$
V(t) \leq \mu^{N_{s}\left(t_{0}, t\right)+1} e^{R\left(t_{0}, t\right)} V_{1}\left(t_{0}\right),
$$

where $R$ is the function defined in (9). Next, using (10), we deduce that

$$
V(t) \leq e^{\ln (\mu) \beta_{*}\left(t-t_{0}\right)+\ln (\mu)\left(\eta_{2}+1\right)} e^{-\alpha_{*}\left(t-t_{0}\right)+\eta_{1}} V_{1}\left(t_{0}\right) .
$$

We deduce from $C_{1}$ that

$$
\gamma_{1}|x(t)|^{2} \leq V(t) \leq e^{\left(\ln (\mu) \beta_{*}-\alpha_{*}\right)\left(t-t_{0}\right)} e^{\eta_{1}+\ln (\mu)\left(\eta_{2}+1\right)} \gamma_{2}\left|x_{t_{0}}\right|_{W}^{2} .
$$

Clearly, the same inequalities are satisfied for $t_{0} \leq t$ such that there is $k \in \mathbb{N}$ such that $t_{0} \in\left[t_{k}, t_{k+1}\right)$ and $t \in\left[t_{k}, t_{k+1}\right)$. This allows us to conclude.

\section{REFERENCES}

[1] W. P. M. H. Heemels, A. R. Teel, N. van de Wouw, and D. Nešić, "Networked control systems with communication constraints: Tradeoffs between transmission intervals, delays and performance," IEEE Transactions on Automatic control, vol. 55, no. 8, pp. 1781-1796, 2010.

[2] X. M. Sun, X. Wang, and Y. Tan, "Absolutely exponential stability and temperature control for gas chromatograph system under dwell time switching techniques," IEEE Transactions on Cybernetics, vol. 46, no. 6, pp. 1301-1310, 2016.

[3] Y. Tang, H. Gao, W. Zhang, and J. Kurths, "Leader-following consensus of a class of stochastic delayed multi-agent systems with partial mixed impulses," Automatica, vol. 53, pp. 346-354, 2015.

[4] X. Wu, Y. Tang, and W. Zhang, "Stability analysis of stochastic delayed systems with an application to multi-agent systems," Automatica, vol. 61, no. 12, pp. 4143-4149, 2016.

[5] F. Mazenc and D. Normand-Cyrot, "Reduction model approach for linear systems with sampled delayed inputs," IEEE Transactions on Automatic Control, vol. 58, no. 5, pp. 1263-1268, 2013.

[6] T. Müller, M. Lauk, and M. Reinhard, "Estimation of delay times in biological systems," Annals of biomedical engineering, vol. 31, no. 11, pp. 1423-1439, 2003.

[7] M. Hong, C. Lin, and B. Shiu, "Stabilizing network control for pneumatic systems with time-delays," Mechatronics, vol. 19, no. 3, pp. 399-409, 2009.

[8] K. Gu, J. Chen, and V. L. Kharitonov, Stability of time-delay systems. Springer Science and Business Media, 2003.

[9] P. G. Park, "A delay-dependent stability criterion for systems with uncertain time-invariant delays," IEEE Transactions on Automatic Control, vol. 44, no. 4, pp. 876-877, 1999.

[10] S. Xu and J. Lam, "Improved delay-dependent stability criteria for time-delay systems," IEEE Transactions on Automatic Control, vol. 50, no. 3, pp. 384-387, 2005.

[11] A. Y. Aleksandrov, G. D. Hu, and A. P. Zhabko, "Delay-independent stability conditions for some classes of nonlinear systems," IEEE Transactions on Automatic Control, vol. 59, no. 8, pp. 2209-2214, 2014.

[12] H. R. Karimi and H. Gao, "Mixed $\mathrm{H}_{2} / \mathrm{H}_{\infty}$ output-feedback control of second-order neutral systems with time-varying state and input delays," ISA transactions, vol. 47, no. 3, pp. 311-324, 2008.

[13] H. R. Karimi, P. Maralani, B. Lohmann, and B. Moshiri, "H $\mathrm{H}_{\infty}$ control of parameter-dependent state-delayed systems using polynomial parameter-dependent quadratic functions," International Journal of Control, vol. 78, no. 4, pp. 254-263, 2005.

[14] Z. Li, H. Gao, and H. R. Karimi, "Stability analysis and $\mathrm{H}_{\infty}$ controller synthesis of discrete-time switched systems with time delay," Systems \& Control Letters, vol. 66, pp. 85-93, 2014.

[15] H. R. Karimi, N. A. Duffie, and S. Dashkovskiy, "Local capacity $\mathrm{H}_{\infty}$ control for production networks of autonomous work systems with time-varying delays," IEEE Transactions on Automation Science and Engineering, vol. 7, no. 4, pp. 849-857, 2010.

[16] I. V. Medvedeva and A. P. Zhabko, "Synthesis of razumikhin and lyapunov-krasovskii approaches to stability analysis of time-delay systems," Automatica, vol. 51, pp. 372-377, 2015. 
[17] Y. Wei, J. Qiu, H. R. Karimi, and M. Wang, "New results on $H_{\infty}$ dynamic output feedback control for markovian jump systems with time-varying delay and defective mode information," Optimal Control, Applications and Methods, vol. 35, no. 6, pp. 656-675, 2014.

[18] Y. Wei, J. Qiu, H. R. Karimi, and W. Ji, "A novel memory filtering design for semi-markovian jump time-delay systems," IEEE Transactions on Systems, Man, and Cybernetics: Systems, vol. 47, no. 8, pp. 2034-2047, 2017.

[19] X. Zhao, H. Yang, W. Xia, and X. Wang, "Adaptive fuzzy hierarchical sliding-mode control for a class of mimo nonlinear time-delay systems with input saturation,” IEEE Transactions on Fuzzy Systems, vol. 25, no. 5, pp. 1062-1077, 2017.

[20] H. Wang, X. Liu, K. Liu, and H. R. Karimi, "Approximation-based adaptive fuzzy tracking control for a class of nonstrict-feedback stochastic nonlinear time-delay systems," IEEE Transactions on Fuzzy Systems, vol. 23, no. 5, pp. 1746-1760, 2015.

[21] H. Wang, P. X. Liu, and P. Shi, "Observer-based fuzzy adaptive output-feedback control of stochastic nonlinear multiple time-delay systems," IEEE Transactions on Cybernetics, 2017.

[22] H. R. Karimi, M. Zapateiro, and N. Luo, "Stability analysis and control synthesis of neutral systems with time-varying delays and nonlinear uncertainties," Chaos, Solitons \& Fractals, vol. 42, no. 1, pp. 595-603, 2009.

[23] H. Gao, X. Meng, and T. Chen, "Stabilization of networked control systems with a new delay characterization," IEEE Transactions on Automatic Control, vol. 53, no. 9, pp. 2142-2148, 2008.

[24] Y. Tang, X. Xing, H. R. Karimi, L. Kocarev, and J. Kurths, "Tracking control of networked multi-agent systems under new characterizations of impulses and its applications in robotic systems," IEEE Transactions on Industrial Electronics, vol. 63, no. 2, pp. 1299-1307, 2016.

[25] B. Xiao, S. Yin, and H. Gao, "Tracking control of robotic manipulators with uncertain kinematics and dynamics," IEEE Transactions on Industrial Electronics, vol. 63, no. 10, pp. 6439-6449, 2016.

[26] H. Wang, H. R. Karimi, P. X. Liu, and H. Yang, "Adaptive neural control of nonlinear systems with unknown control directions and input dead-zone," IEEE Transactions on Systems, Man, and Cybernetics: Systems, 2017.

[27] B. Xiao and S. Yin, "Velocity-free fault tolerant and uncertainty attenuation control for a class of nonlinear systems," IEEE Transactions on Industrial Electronics, vol. 63, no. 7, pp. 4400-4411, 2016.

[28] M. Blanke and J. Schröder, Diagnosis and fault-tolerant control. Springer, 2006, vol. 691.

[29] B. Xiao, Q. Hu, and P. Shi, "Attitude stabilization of spacecraft under actuator saturation and partial loss of control effectiveness," IEEE Transactions on Control System Technology, vol. 21, no. 6, pp. 2251-2263, 2013.

[30] X. M. Sun, G. Liu, D. Rees, and W. Wang, "Stability of systems with controller failure and time-varying delay," IEEE Transactions on Automatic Control, vol. 53, no. 10, pp. 2391-2396, 2008.

[31] X. M. Sun, G. Liu, W. Wang, and D. Rees, "Stability analysis for networked control systems based on average dwell time method," International Journal of Robust and Nonlinear Control, vol. 20, no. 15, pp. 1774-1784, 2010.

[32] Z. H. Pang, G. P. Liu, D. Zhou, and D. Sun, "Data-based predictive control for networked nonlinear systems with network-induced delay and packet dropout," IEEE Transactions on Industrial Electronics, vol. 63, no. 2, pp. 1249-1257, 2016.

[33] M. T. Hale, A. Nedić, and M. Egerstedt, "Cloud-based centralized/decentralized multi-agent optimization with communication delays," Proceedings of IEEE 54th Annual Conference on Decision and Control (CDC), pp. 700-705, 2016.

[34] D. Liberzon, Switching in systems and control. Boston: Birkhauser, 2003.

[35] H. Lin and P. Antsaklis, "Stability and stabilizability of switched linear systems: A survey of recent results," IEEE Transactions on Automatic Control, vol. 54, no. 2, pp. 308-322, 2009.

[36] S. Y. Caliskan, H. Ozbay, and S. I. Niculescu, "Dwell-time computation for stability of switched systems with time delays," IET Control Theory and Applications, vol. 7, no. 10, pp. 1422-1428, 2013.

[37] X. M. Sun, G. P. Liu, D. Rees, and W. Wang, "Delay-dependent stability for discrete systems with large delay sequence based on switching techniques," Automatica, vol. 44, no. 11, pp. 2902-2908, 2008.

[38] X. M. Sun, G. Liu, W. Wang, and D. Rees, "Stability analysis for systems with large delay period: A switching method," International Journal of Innovative Computing, Information and Control, vol. 8, no. 6, pp. 4235-4247, 2012.

[39] X. M. Sun, S. Du, P. Shi, W. Wang, and L. Wang, "Input-to-state stability for nonlinear systems with large delay periods based on switching techniques," IEEE Transactions on circuits and systems I: regular papers, vol. 61, no. 6, pp. 1789-1800, 2014.

[40] E. Fridman, M. Dambrine, and N. Yeganefar, "On input-to-state stability of systems with time-delay: A matrix inequalities approach," IEEE Transactions on Systems, Man, and Cybernetics: Systems, vol. 44, pp. 2364-2369, 2008. 